\title{
A novel methodology for in vivo endoscopic phenotyping of colorectal cancer based on real-time analysis of the mucosal lipidome: a prospective observational study of the iKnife
}

\author{
James Alexander ${ }^{1} \cdot$ Louise Gildea $^{1} \cdot$ Julia Balog $^{2} \cdot$ Abigail Speller $^{3} \cdot$ \\ James McKenzie ${ }^{1}$ - Laura Muirhead ${ }^{1}$ - Alasdair $\operatorname{Scott}^{1} \cdot$ Christos Kontovounisios $^{5}$. \\ Shanawaz Rasheed $^{5} \cdot$ Julian Teare $^{1} \cdot$ Jonathan Hoare $^{4} \cdot$ Kirill Veselkov $^{1}$. \\ Robert Goldin $^{3} \cdot$ Paris Tekkis $^{5} \cdot$ Ara Darzi $^{1} \cdot$ Jeremy Nicholson $^{1} \cdot$ James Kinross $^{1}$ • \\ Zoltan Takats ${ }^{1}$
}

Received: 24 March 2016/ Accepted: 12 July 2016/Published online: 8 August 2016

(C) The Author(s) 2016. This article is published with open access at Springerlink.com

\begin{abstract}
Background This pilot study assessed the diagnostic accuracy of rapid evaporative ionization mass spectrometry (REIMS) in colorectal cancer (CRC) and colonic adenomas.

Methods Patients undergoing elective surgical resection for CRC were recruited at St. Mary's Hospital London and The Royal Marsden Hospital, UK. Ex vivo analysis was performed using a standard electrosurgery handpiece with aspiration of the electrosurgical aerosol to a Xevo G2-S iKnife QTof mass spectrometer (Waters Corporation). Histological examination was performed for validation purposes. Multivariate analysis was performed using principal component analysis and linear discriminant analysis in Matlab 2015a (Mathworks, Natick, MA). A modified
\end{abstract}

Presented at the SAGES 2016 Annual Meeting, March 16-19, 2016, Boston, Massachusetts

Electronic supplementary material The online version of this article (doi:10.1007/s00464-016-5121-5) contains supplementary material, which is available to authorized users.

James Kinross

j.kinross@imperial.ac.uk

1 Department of Surgery and Cancer, Imperial College

London, St. Mary's Hospital, Praed Street,

London NW1 1SQ, UK

2 Waters Corporation, Wilmslow, UK

3 Department of Histopathology, Imperial College London, London, UK

4 Department of Gastroenterology, Imperial Healthcare NHS Trust, London, UK

5 Department of Colorectal Surgery, Royal Marsden Hospital NHS Trust, London, UK
REIMS endoscopic snare was developed (Medwork) and used prospectively in five patients to assess its feasibility during hot snare polypectomy.

Results Twenty-eight patients were recruited (12 males, median age 71, range 35-89). REIMS was able to reliably distinguish between cancer and normal adjacent mucosa (NAM) (AUC 0.96) and between NAM and adenoma (AUC 0.99). It had an overall accuracy of $94.4 \%$ for the detection of cancer versus adenoma and an adenoma sensitivity of $78.6 \%$ and specificity of $97.3 \%$ (AUC 0.99) versus cancer. Long-chain phosphatidylserines (e.g., PS 22:0) and bacterial phosphatidylglycerols were over-expressed on cancer samples, while NAM was defined by raised plasmalogens and triacylglycerols expression and adenomas demonstrated an over-expression of ceramides. REIMS was able to classify samples according to tumor differentiation, tumor budding, lymphovascular invasion, extramural vascular invasion and lymph node micrometastases (AUC's 0.88, 0.87, 0.83, 0.81 and 0.81, respectively). During endoscopic deployment, colonoscopic REIMS was able to detect target lipid species such as ceramides during hot snare polypectomy.

Conclusion REIMS demonstrates high diagnostic accuracy for tumor type and for established histological features of poor prognostic outcome in CRC based on a multivariate analysis of the mucosal lipidome. REIMS could augment endoscopic and imaging technologies for precision phenotyping of colorectal cancer.

Keywords Rapid evaporative ionization mass spectrometry $\cdot$ Colorectal cancer $\cdot$ Metabolic

Precision medicine aims to improve patient outcomes, by coupling established clinical-pathological indexes with 
state-of-the-art molecular profiling to create diagnostic, prognostic and therapeutic strategies precisely tailored to each patient's requirements $[1,2]$. There is an unmet need for precision polyp and cancer phenotyping for stratification of surgical and neoadjuvant therapies in the treatment of colorectal cancer. In the management of complex rectal polyps and early rectal cancer, the decision to proceed to a radical resection is largely based on a combination of imaging and clinical assessment. Current protocols for risk stratification of polyps are based on subjective descriptions of polyp morphology such as the Paris classification although these are not highly reproducible [3] and histopathological analysis is also subjective.

Rapid evaporative ionization mass spectrometry (REIMS) is a novel technique based on the mass spectrometric analysis of the aerosol produced during electrosurgical diathermy to provide tissue specific diagnostics based on lipid profiles [4-6]. This technology is able to provide rapid metabolic phenotyping of rectal and colonic cancers using the mass spectrometric lipid signatures for the identification of unknown tissue features intra-operatively with correct classification efficiencies exceeding $92 \%$ in real-time and $97 \%$ for ex vivo analyses [7]. Recently, we have demonstrated that this technology can be deployed endoluminally in the upper and lower GI tract; however, its true application in colonoscopy is not yet known [8]. As a follow-up to our earlier work, in this pilot study, we assessed the accuracy of endoscopic REIMS for the classification of both benign and neoplastic colorectal lesions and to provide chemical information associated with poor prognostic histological features commonly assessed in colorectal cancer. The aim was to assess the feasibility of this technology to provide information of clinical utility, and this was achieved using an ex vivo analysis of tissue samples, which permits the creation of a spectral database for prospective use. The secondary aim was to further demonstrate that in vivo applications of endoscopic REIMS could be scaled and reliably reproduced. A prototype was therefore developed for deployment of REIMS during colonoscopy.

\section{Methods}

\section{Patient recruitment and sampling}

Between January and March 2015, 28 patients undergoing elective surgical treatment for colorectal cancer were prospectively recruited at two London teaching hospitals (St Mary's Hospital and The Royal Marsden Hospital). Full local ethical approval was obtained (14/EE/0024). Inclusion criteria were adult patients with a suspected primary colorectal cancer undergoing elective surgical resection by either open or laparoscopic surgery. Patients who had undergone neoadjuvant chemoradiotherapy were included. Patients unable to provide consent or those undergoing emergency surgery were excluded, as were patients with tumors associated with hereditary conditions such as Familial Adenomatous Polyposis, Hereditary Nonpolyposis Colorectal Cancer or inflammatory bowel disease. The primary endpoint of the study was the diagnosis of colorectal cancer, as differentiated from adenoma and normal adjacent mucosa (NAM). Secondary endpoints were the diagnosis of histological prognostic biomarkers: TNM stage, tumor differentiation, lymphovascular invasion, extramural vascular invasion and tumor budding and complete pathological response (cPR) to neoadjuvant therapy. Following resection, surgical specimens were immediately transported fresh to the histopathology department by a member of the research team. The specimen was opened by a trained histopathologist, and fresh biopsies were cut from the tumor and normal adjacent mucosa at $10 \mathrm{~cm}$ from the lesion.

\section{Ex vivo analysis}

The fresh biopsies were analyzed ex vivo with an electrosurgery hand-piece using monopolar diathermy in cutting mode (ValleylabTM) with aspiration of the electrosurgical aerosol to a Xevo G2-S iKnife Q-Tof mass spectrometer (Waters Corporation). Following formalin fixation, the biopsies underwent histological examination by two trained histopathologists (AS \& RG) for validation purposes. Full classification was performed according to the minimum dataset recommendations of the Royal College of Pathologists [9].

\section{Statistical analysis}

Four high-quality mass spectra were obtained per sample. REIMS raw spectral data were converted to imzML format (MSConvert) and imported into Matlab R2015a (Mathworks, Natick, MA) for processing within a toolbox designed in-house for interrogation of clinical mass spectral datasets. The compiled spectra were normalized to their total ion count to reduce the impact of variation in overall signal intensity unrelated to spectral pattern and then $\log$ transformed to account for large variability differences between small and large peaks across mass spectra [10]. Discriminating models were then subjected to dimensionality reduction via principal components analysis to reduce the number of observed variables into a smaller number of orthogonal principal components, which account for the majority of the variance in the dataset. The first 30 principal components were kept for further analysis. Linear discriminant analysis was then used to 
maximize the separation between different classes, and the resulting LDA space was used for classification of spectra from unknown tissue samples using Mahalanobis distance calculations. All mass spectral data belonging to one patient were left out of the sample set, and a new model was calculated using the remaining data. The withheld data were projected into the new model and classified as one tissue type using Mahalanobis distances calculations, performed between the unknown sample point and calculated class centers. This process was repeated for each individual patient. From this, the diagnostic sensitivity and specificity could be calculated as were ROC curves to provide a crossvalidated score that describes the clinical utility of the model. To identify statistically significant peaks responsible for prediction into each class, for each cross-validation iteration, analysis of variance (ANOVA) was employed to only select the features most statistically significant ( $p$ value $<0.05$ ) for PCA-LDA classification into each group. Exact $m / z$ values were retrieved from the raw data, and these values were used for compound identification via database search (Metlin).

\section{Results}

Twenty-eight patients were recruited for the ex vivo phase of the study (12 males, 16 females). Two patients were subsequently excluded from the final analysis: In one case, it was not possible to acquire a tissue sample for REIMS analysis because the tumor was not sufficient (a minimum tumor size of $10 \mathrm{~mm}$ diameter was required by the histopathologist for research sampling); in the other case, it was not possible to obtain adequate spectra from the REIMS instrument, and the signal-to-noise ratio was too low. Patient demographics and histological classification of the remaining cases are shown in Table 1 . Two patients had an adenoma, fifteen patients had adenocarcinoma, eight patients had mucinous adenocarcinoma, and one patient had a gastrointestinal stromal tumor (GIST).

\section{Diagnostic accuracy and histological biomarker association}

Representative REIMS spectra from NAM, a cancer and an adenoma can be seen in Fig. 1. REIMS was able to reliably distinguish between NAM and cancer (AUC 0.96) and between NAM and adenoma (AUC 0.99). It had an overall accuracy of $94.4 \%$ for the detection of cancer versus adenoma (Table 2) and an adenoma sensitivity of $78.6 \%$ and specificity of $97.3 \%$ (AUC 0.99) versus cancer (Fig. 2). Univariate analysis of target metabolites was performed based on results from the loadings data to
Table 1 Patient demographics and histopathological classification from ex vivo analysis

\begin{tabular}{|c|c|}
\hline Male/female & $11 / 15$ \\
\hline Median age (range) & $71(35-89)$ \\
\hline Primary/recurrence & $24 / 2$ \\
\hline Neoadjuvant treatment & 4 \\
\hline \multicolumn{2}{|l|}{ Site of lesion } \\
\hline Cecum & 6 \\
\hline Ascending colon & 1 \\
\hline Transverse colon & 2 \\
\hline Descending colon & 0 \\
\hline Sigmoid colon & 7 \\
\hline Rectum & 10 \\
\hline \multicolumn{2}{|l|}{ Histopathology } \\
\hline Adenoma & $2^{\mathrm{a}}$ \\
\hline Adenocarcinoma & 15 \\
\hline Mucinous adenocarcinoma & 8 \\
\hline Gastrointestinal stromal tumor (GIST) & 1 \\
\hline \multicolumn{2}{|l|}{ Tumor differentiation } \\
\hline Well & 1 \\
\hline Moderate & 16 \\
\hline Poor & 5 \\
\hline Not applicable & $4^{\mathrm{b}}$ \\
\hline \multicolumn{2}{|l|}{ T stage } \\
\hline T0 & $4^{\mathrm{b}}$ \\
\hline $\mathrm{T} 1$ & 1 \\
\hline $\mathrm{T} 2$ & 5 \\
\hline $\mathrm{T} 3$ & 11 \\
\hline $\mathrm{T} 4$ & 5 \\
\hline \multicolumn{2}{|l|}{$\mathrm{N}$ stage } \\
\hline No & 19 \\
\hline N1 & 6 \\
\hline $\mathrm{N} 2$ & 1 \\
\hline \multicolumn{2}{|l|}{ M stage } \\
\hline M0 & 25 \\
\hline M1 & 1 \\
\hline Extramural vascular invasion & 7 \\
\hline Lymphovascular invasion & 9 \\
\hline Tumor budding & 16 \\
\hline
\end{tabular}

a Benign histology but with suspicious features on preoperative imaging

b In addition to the two patients' tumors which were histologically adenomas, two patients had no viable tumor remaining after complete pathological response to neoadjuvant therapy

confirm their statistical contribution to the model and to identify descriptive metabolites responsible for class description (Table 3). This confirms that lipid expression in cancer is dynamic, and quantitative changes in target lipids can be identified between pathological disease states. Cancer has an over-expression of long-chain phosphatidylserines (e.g., PS 22:0) and bacterial 
Table 2 Summary diagnostic and sensitivity data from the multivariate models

\begin{tabular}{|c|c|c|c|c|c|c|c|}
\hline & $\begin{array}{l}\text { Spectra } \\
n=\end{array}$ & Accuracy & $\begin{array}{l}\text { True } \\
\text { positive }\end{array}$ & $\begin{array}{l}\text { True } \\
\text { negative }\end{array}$ & $\begin{array}{l}\text { False } \\
\text { positive }\end{array}$ & $\begin{array}{l}\text { False } \\
\text { negative }\end{array}$ & AUC \\
\hline \multicolumn{8}{|l|}{ Diagnostic markers all } \\
\hline Cancer versus NAM & 220 & $90.5 \%$ & $86.7 \%$ & $92.4 \%$ & $13.3 \%$ & $7.6 \%$ & 0.96 \\
\hline Cancer versus adenoma & 89 & $94.4 \%$ & $78.6 \%$ & $97.3 \%$ & $2.7 \%$ & $21.4 \%$ & 0.99 \\
\hline Adenoma versus NAM & 159 & $97.5 \%$ & $85.7 \%$ & $98.6 \%$ & $1.4 \%$ & $14.3 \%$ & 0.99 \\
\hline $\begin{array}{l}\text { Histological subtype (mucinous vs. } \\
\text { adenocarcinoma) }\end{array}$ & 75 & $90 \%$ & $94.2 \%$ & $83.3 \%$ & $16.7 \%$ & $5.8 \%$ & 0.96 \\
\hline \multicolumn{8}{|l|}{ Prognostic performance-whole model } \\
\hline Tumor differentiation (mod vs. poor) & 183 & $83.1 \%$ & $68.3 \%$ & $87.3 \%$ & $12.7 \%$ & $31.7 \%$ & 0.88 \\
\hline Tumor budding & 234 & $78.2 \%$ & $80.6 \%$ & $74.4 \%$ & $25.6 \%$ & $19.4 \%$ & 0.87 \\
\hline LVI & 234 & $73.9 \%$ & $71.6 \%$ & $75.3 \%$ & $24.7 \%$ & $28.4 \%$ & 0.83 \\
\hline EMVI & 234 & $73.5 \%$ & $65.3 \%$ & $77.2 \%$ & $22.8 \%$ & $34.7 \%$ & 0.81 \\
\hline +ve nodes & 234 & $77.4 \%$ & $69.0 \%$ & $81.0 \%$ & $19.0 \%$ & $31.0 \%$ & 0.81 \\
\hline \multicolumn{8}{|l|}{ Rectal cancer prognostic factors } \\
\hline Differentiation (mod vs. poor) & 84 & $94.4 \%$ & $78.6 \%$ & $98.2 \%$ & $1.8 \%$ & $21.4 \%$ & 0.99 \\
\hline Tumor budding & 84 & $84.5 \%$ & $88.1 \%$ & $70.6 \%$ & $29.4 \%$ & $11.9 \%$ & 0.82 \\
\hline LVI & 84 & $71.4 \%$ & $72.4 \%$ & $30.8 \%$ & $69.2 \%$ & $27.6 \%$ & 0.75 \\
\hline EMVI & 84 & $96.4 \%$ & $85.7 \%$ & $98.6 \%$ & $14.3 \%$ & $1.4 \%$ & 0.98 \\
\hline +ve nodes & 84 & $92.9 \%$ & $83.3 \%$ & $94.4 \%$ & $5.6 \%$ & $16.7 \%$ & 0.92 \\
\hline LCRT versus none & 75 & $96 \%$ & $95.7 \%$ & $96.2 \%$ & $3.8 \%$ & $4.3 \%$ & 0.99 \\
\hline $\mathrm{cPR}$ versus NAM & 52 & $100 \%$ & $100 \%$ & $100 \%$ & $0 \%$ & $0 \%$ & 1 \\
\hline
\end{tabular}

$A U C$ area under curve, NAM normal adjacent mucosa, Mod moderate, LCRT long-course chemoradiotherapy, $c P R$ complete pathological response

Table 3 Summary putative metabolite IDs for cancer, adenoma and normal colonic mucosa, their $\mathrm{m} / \mathrm{z}$ and statistical significance ( $p$ value)

\begin{tabular}{|c|c|c|c|c|c|}
\hline$m / z$ & $\begin{array}{l}\text { Putative molecular } \\
\text { structure }\end{array}$ & $p$ value & Cancer & Adenoma & Norma \\
\hline 633.465 & PA (31:0) & 0.0001 & & & \\
\hline 642.495 & GlcCer (30:1) & $2.47 \mathrm{E}-12$ & & & \\
\hline 653.495 & 1,2-DG (36:3) & 0.0002 & & & \\
\hline 673.485 & PA (34:0) & $3.33 E-16$ & & & \\
\hline 699.505 & PA & $1.12 \mathrm{E}-07$ & & & \\
\hline 702.625 & Cer (D18:0/H24:0) & $2.28 \mathrm{E}-11$ & & & \\
\hline 710.485 & PE (34:4) & $6.73 \mathrm{E}-08$ & & & \\
\hline 718.615 & $\begin{array}{l}\text { Cer } \\
(\mathrm{T} 18: 0 / 24: 0(2 \mathrm{OH}))\end{array}$ & $9.30 \mathrm{E}-10$ & & & \\
\hline 775.545 & PG (36:1) & 0.0001 & & & \\
\hline 797.535 & PG (38:4) & 0.004 & & & \\
\hline 864.675 & Plasmalogen & 0.0004 & & & \\
\hline 880.665 & PS (43:4) & 1.86E-07 & & & \\
\hline 886.565 & PS (44:6) & $1.110 \mathrm{E}-16$ & & & \\
\hline 886.565 & PS (44:8) & 1.110E-16 & & & \\
\hline 888.75 & PS (41:0) & $1.38 \mathrm{E}-12$ & & & \\
\hline 891.725 & TG $(54: 0)$ & $1.63 \mathrm{E}-08$ & & & \\
\hline
\end{tabular}

The array visualization demonstrates if the metabolites were over-expressed (red) or under-expressed (green) in specific histological states of cancer, adenoma or normal associated mucosa 


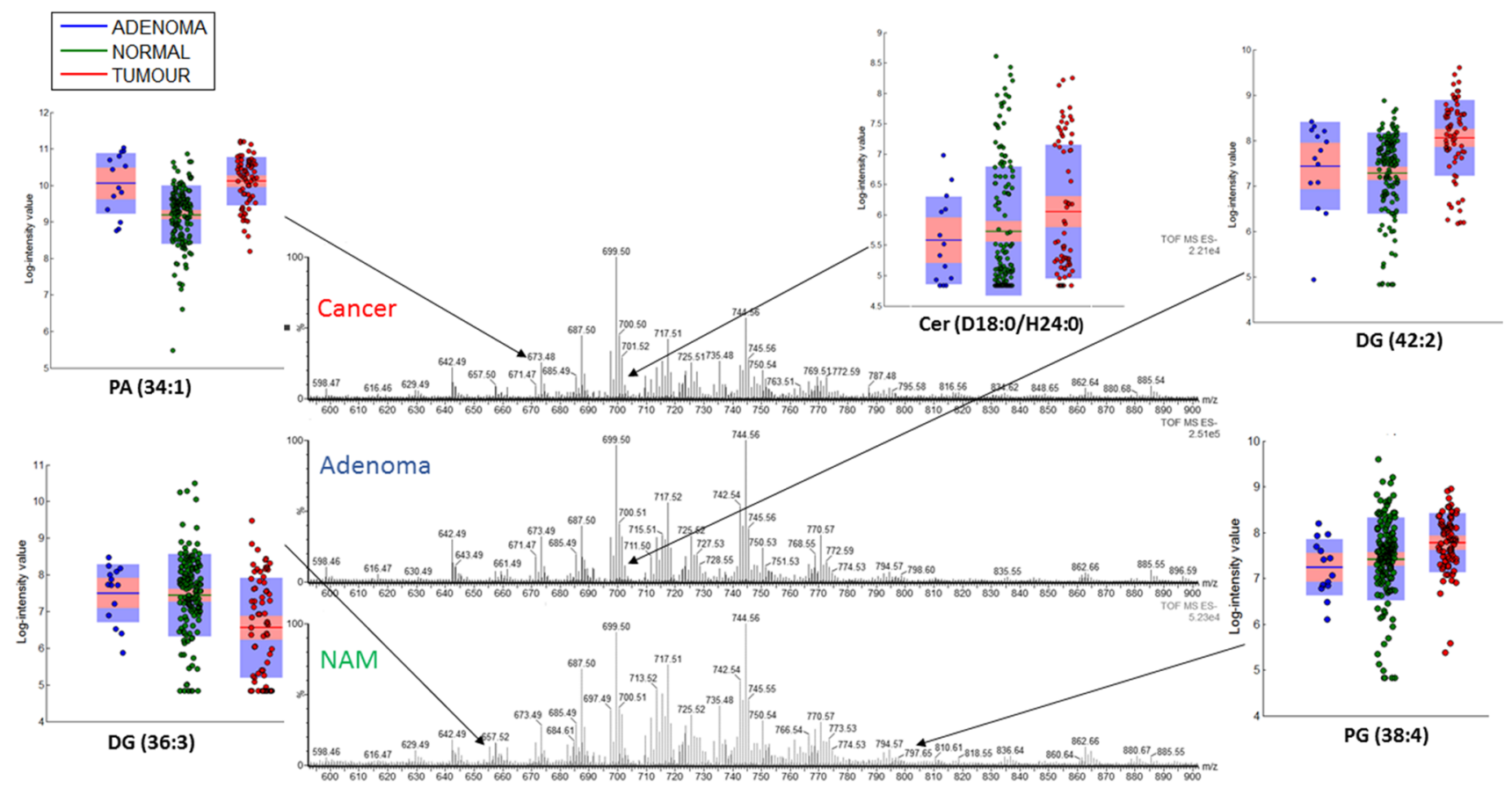

Fig. 1 Example spectra from NAM, malignant and adenomatous tissue; the $600-900 \mathrm{~m} / \mathrm{z}$ range has been selected. Box plots representing statistically significant phospholipid species from the entire sample

phosphatidylglycerols, while healthy mucosa has an overexpression of plasmalogens and triacylglycerols (Fig. 1; Table 3), and adenomas demonstrated an over-expression of ceramides (Fig. 1).

To assess the influence of possible patient confounders on the model's performance, the spectral data were modeled against age and sex, and it was not possible to build a strongly predictive model for either of these factors (Supp. Figure $1 \mathrm{a}$ and $\mathrm{b}$ ). However, males and patients over the age of 70 did demonstrate discrete clustering, suggesting that there may be both age and sex subtle changes detectable in the colonic mucosa. Analysis of anatomical sampling points also provided a weak model; however, rectal samples were distinctly clustered from the remaining colonic specimens. These data suggest that the rectal mucosa harbors a discrete colonic mucosal lipidome that can be chemically identified (Supp. Figure $1 \mathrm{c}$ ).

Using the whole dataset, it was possible to provide information on the histological subtype of tumors. Only one patient in this cohort had a GIST, and although they clustered separately, they were excluded from the analysis. REIMS was able to distinguish between adenocarcinoma and mucinous adenocarcinoma with a high degree of accuracy (Table 2), with an AUC of 0.96 and a sensitivity and specificity of 94.2 and $83.3 \%$, respectively. As an extension, chemical staging models were constructed to establish how the tumor mucosal lipidome evolves with advancing stage. The overall accuracy of the staging set are demonstrated identifying statistically significant quantitative changes in lipid expression between tissue types

model was $74.7 \%$, with stage 0 cancers (Fig. 4D) and stage IV cancers clustering separately, and there was considerable overlap between stages II and III lesions. It was possible to classify all tumor specimens according to the lymphatic involvement (Fig. 3) with an accuracy of $83.5 \%$, although because of the small number of $\mathrm{N} 2$ lesions, the sensitivity and specificity was not recordable. The model for metastatic status had sensitivity for distinguishing metastatic from non-metastatic disease of $25 \%$ (Supp. Figure $1 \mathrm{f}$ ).

During the in vivo resection of polyps or cancers, numerous sample points are obtained during a single surgical REIMS-augmented procedure, and all of these provide important biochemical data that are relevant to the summary histological classification. Therefore, all sampling points were included (both tumor and NAMs) in the analysis of prognosis. Histopathology revealed that seven tumors had lymph node metastases at the time of histopathological assessment (LN). Seven tumors had extramural vascular invasion (EMVI), and sixteen tumors had tumor budding (TB). The overall performance for all patients included in the analysis was moderate; AUCs of REIMS for all samples in the cohort for differentiation, tumor budding, LVI, EMVI and $\mathrm{LN}$ involvement were $0.88,0.87,0.83,0.81$ and 0.81 , respectively (Fig. 3, Table 2). In general, the specificity of the technique was greater than the sensitivity, although both the false-negative and false-positive discovery rate were variable (Table 2). 


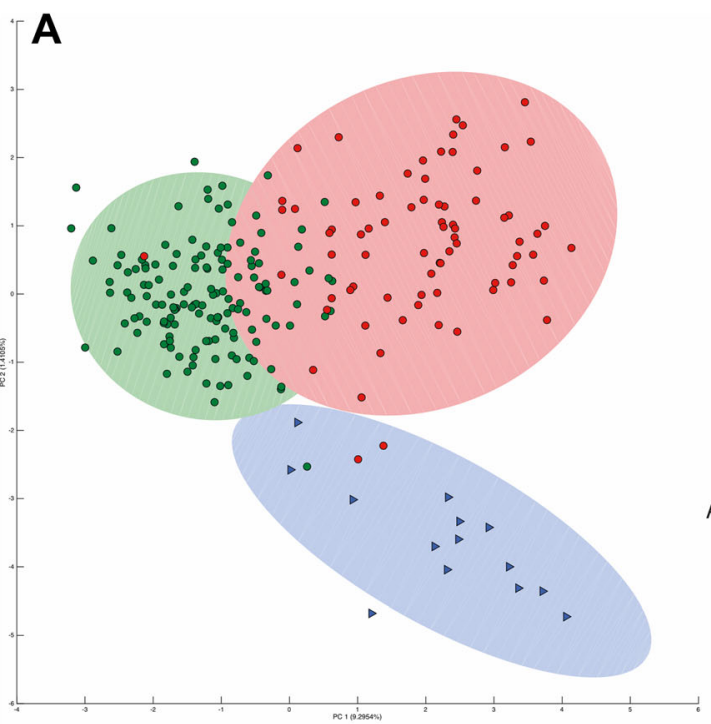

Normal associated mucosa

Cancer

Adenoma
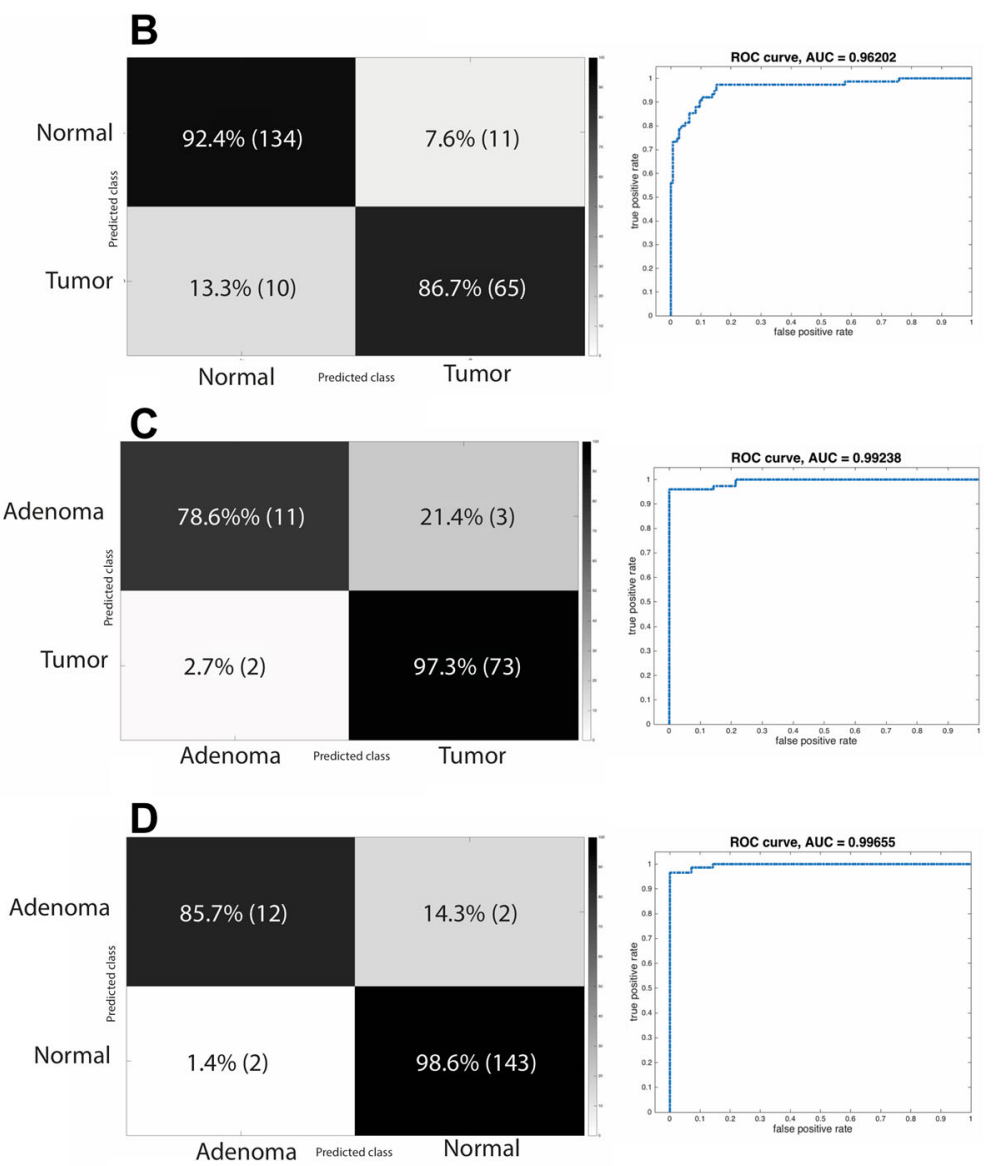

Fig. 2 Summary diagnostic colorectal data analyzed by REIMS. A An LDA scores plot, demonstrating class separation between normal associated mucosa (NAM), cancer and adenomas. The confusion matrices showing the sensitivity and specificity of B NAM versus Tumor C Tumor versus adenoma and D NAM versus adenoma are demonstrated in the corresponding ROC curves
To account for the influence on anatomical lipid expression and the confounding effect of neoadjuvant therapies, rectal cancers were then examined in isolation. Four of these patients had undergone neoadjuvant treatment with long-course chemoradiotherapy (LCRT), and multivariate modeling suggested that this has a persistent influence on the rectal lipid chemistry, as the model was able to robustly differentiate those patients treated with LCRT (Sensitivity $95.7 \%$, specificity $96.2 \%$, AUC 0.9 ). Two patients had no viable tumor remaining after complete pathological response to neoadjuvant therapy, however. Histopathological assessment of these biopsies revealed fibrosis, and REIMS was able to robustly distinguish this from healthy tissue with $100 \%$ accuracy (Table 2).

Routine histological reporting outcomes that serve as established biomarkers of poor prognostic outcome and which are used to guide future therapy were then used as arbitrary outcome measures to determine whether REIMS has the ability to define rectal tumors of poor phenotype. REIMS was able to differentiate tumors according to tumor budding (AUC 0.82), differentiation (moderate vs. poor AUC 0.99), LVI (AUC 0.75), EMVI (AUC 0.98) and nodal micrometastases (AUC 0.92). The histological correlations were stronger in the rectal cohort than in the remaining colonic group suggesting that the lipid analysis has the capacity to identify chemical signatures associated with a poor outcome in patients with established rectal cancer.

\section{In vivo development}

The in vivo endoscopic REIMS system was set up as outlined in Fig. 4. A bespoke modified endoscopic polypectomy snare (Medwork) was developed to allow optimum transfer of snare diathermy aerosol from the colonic lumen. Modifications included a series of fenestrations in the distal snare sheath and an inbuilt side port in the snare handle to facilitate connection to the mass spectrometer (Fig. 4D). A video of the system setup and live data capture can be seen in the supplementary data. During the initial experiments, the spectral data were not 


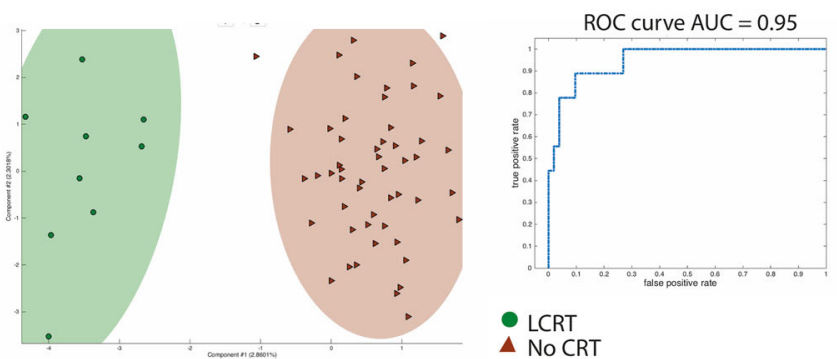

A Neoadjuvant chemoradiotherapy

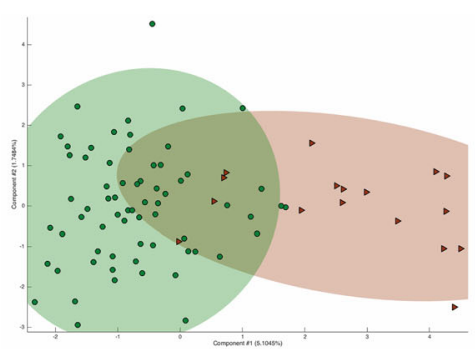

\section{B Tumour budding}
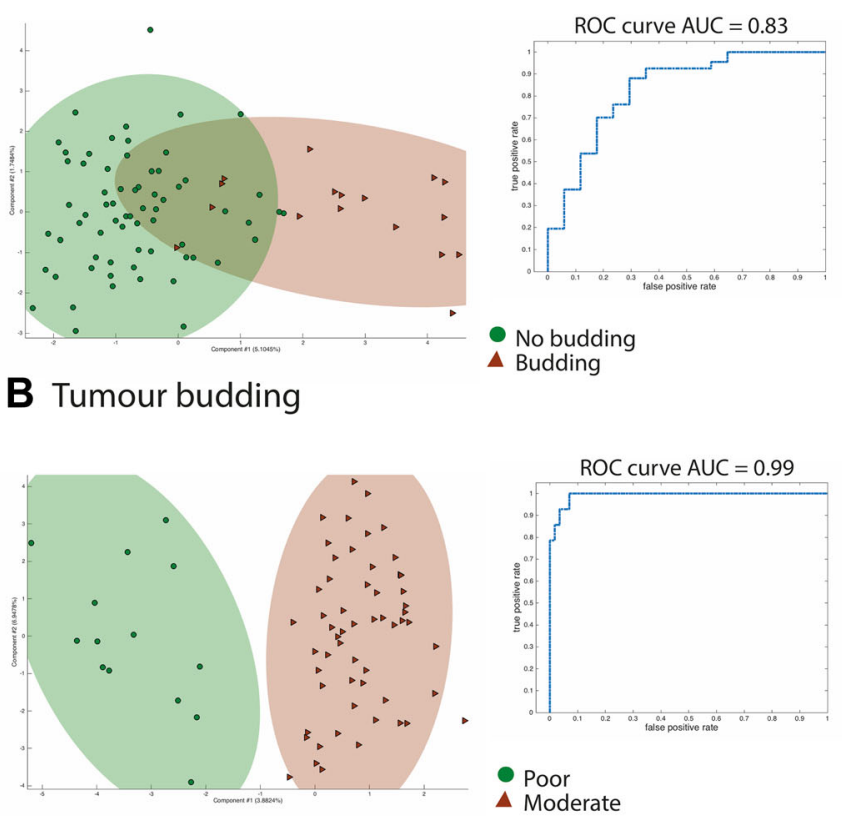

\section{Tumour differentitation}

Fig. 3 Summary LDA scores plots and associated ROC curves for associations with established histopathological biomarkers of poor prognosis. The model was able to identify those patients that had

provided to the endoscopist or clinical team. The system was deployed in vivo in five patients undergoing polypectomy as part of the UK Bowel Cancer Screening Program, and the demographic data for the patients recruited to the in vivo work can be seen in supplementary Table 1. Initial analysis was focused on proof of concept; therefore, the aim was the optimization of data quality, as determined by signal-to-noise ratio. Initial experiments suffered from obstruction of the ion transfer tubing and thus poor quality signals. However, these were overcome, and the snare fenestrations were optimized. An example of the current spectral data quality can be found in the supplementary data (Supp. Figure 3). This demonstrates that target lipid species as identified by the ex vivo analysis are visible within the selected $\mathrm{m} / \mathrm{z}$ charge range, and that they can be captured in near real time. However, it is not currently possible to perform a valid multivariate analysis of its diagnostic strength.
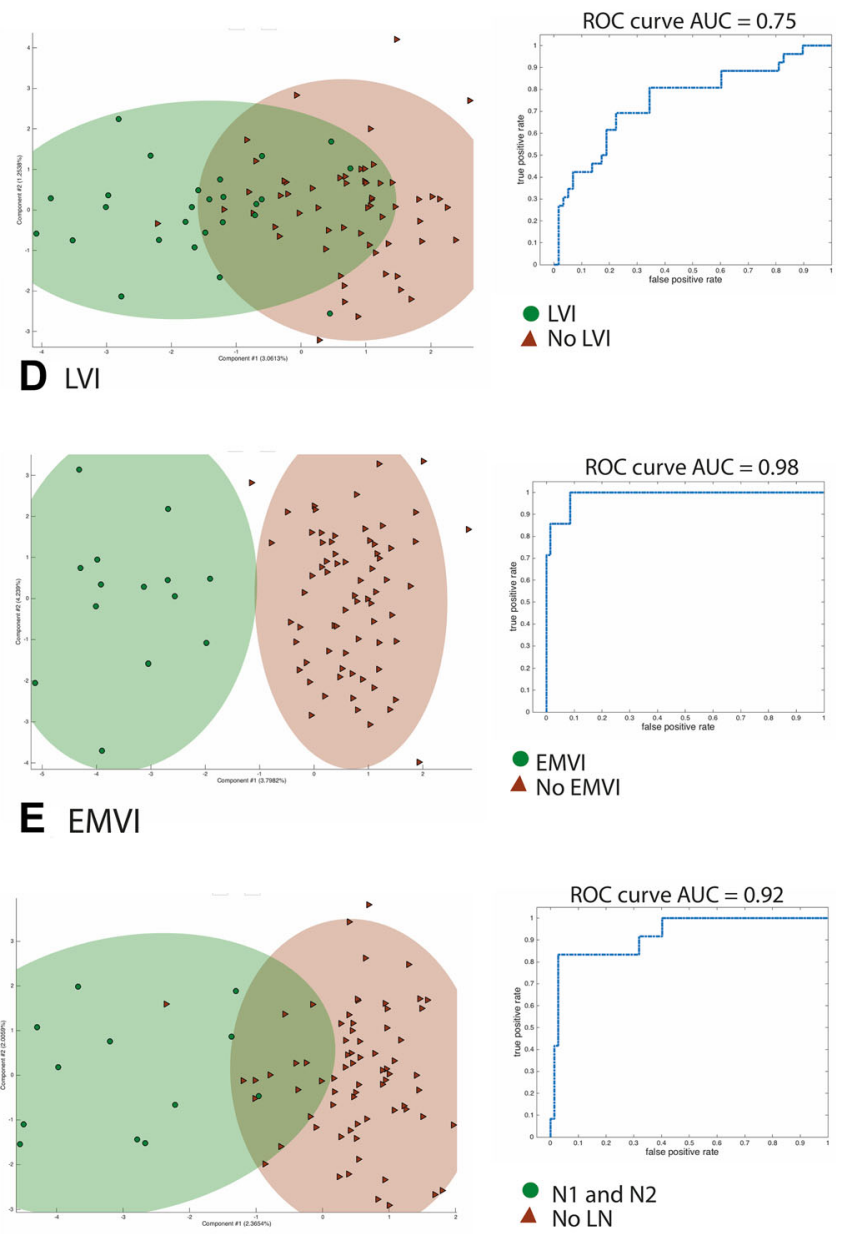

F N1 and N2+ve

undergone neoadjuvant LCRT. It was also able to provide robust models for A tumor budding B differentiation C LVI, D EMVI and E Lymph node micrometastases

\section{Discussion}

Precision surgery for the management of colorectal cancer requires new technologies that can provide real-time, objective data on tumor biology for the stratification of clinical decision-making. The cancer lipidome is rapidly being realized as a key modulator of cancer biology and therapeutic response, and it provides a novel window into tumor biology. It also offers a novel methodology for in vivo analysis as it can be assessed in near real time. Some tumors demonstrate a lipogenic phenotype, as membrane lipids are rapidly synthesized in tumor cells with a high turnover. Indeed, cancer cells alter lipid metabolism pathways in order to optimize de novo lipid/ fatty acid output [11]. Our previous work on colorectal cancer (CRC) metabolism has confirmed markedly altered lipid patterns in healthy compared to cancerous colorectal epithelium [12-15]. In addition, recent cell-based work has 


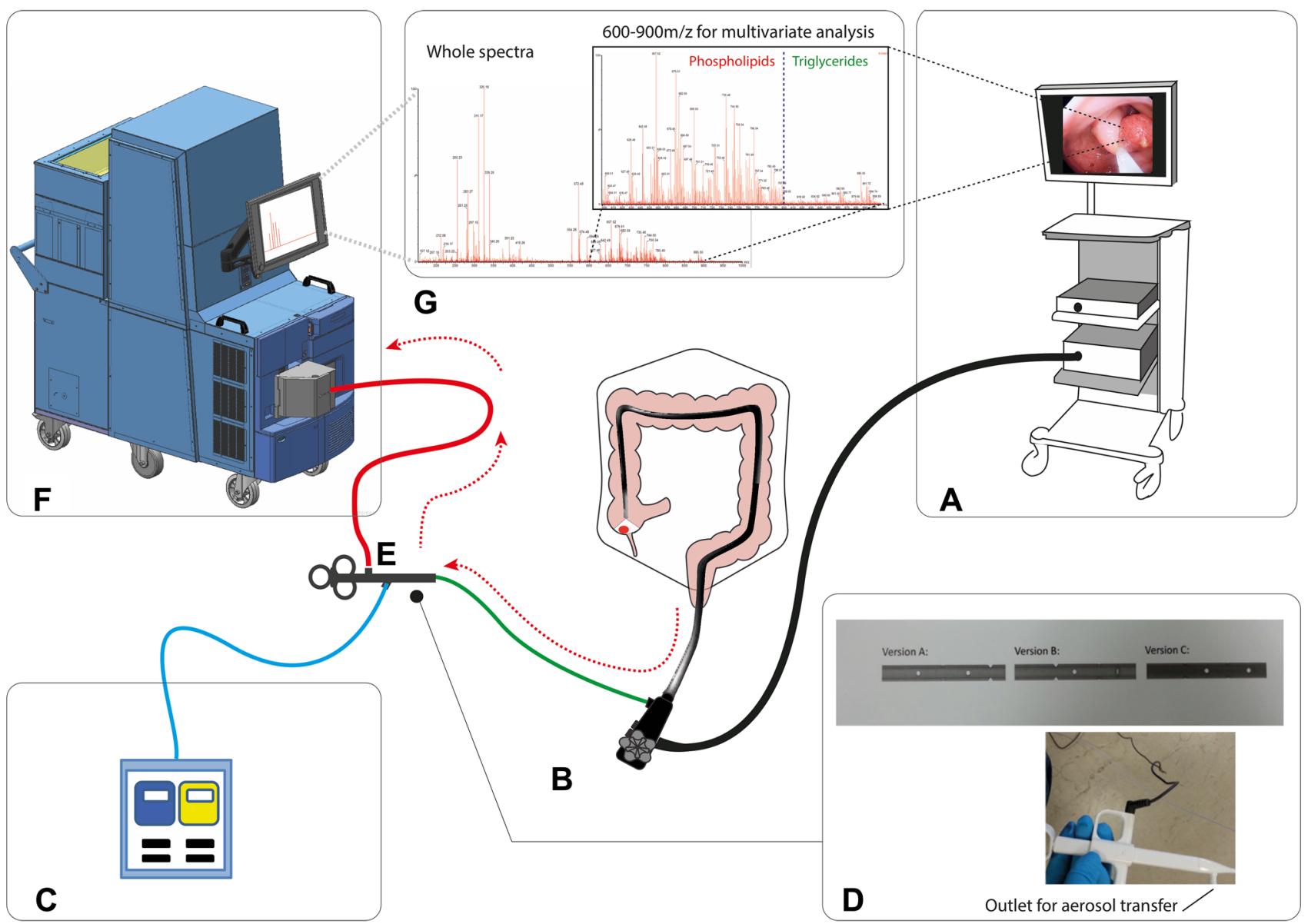

Fig. 4 Summary overview of the iEndoscope platform. This technology requires minimal deviation from a standard endoscopic set up with A A typical stack and B Any commercially available endoscope. C A standard electrosurgical generator is deployed during 'hot' endoscopic resection. D We have created a modified snare with a fenestrated distal sheath for improved efficiency of smoke aspiration. This was developed in three variants. It has a working channel for aspiration directly to $\mathbf{F}$ a mobile mass spectrometer, with a self contained spectral database sited in the endoscopy suite. Data are in

implicated lipids as critical regulators of cancer not only structurally (for cell membrane synthesis and stabilization) but also in cell-signaling and cross talk between cancer cells and the cancer-adjacent stroma [11].

This is the first study to apply REIMS to a well-phenotyped, prospectively recruited colorectal cancer patient cohort. The aim of this study was to perform a validation experiment to determine the diagnostic potential of REIMS in colorectal cancer and to investigate the potential of REIMS as a point of care, prognostic stratification tool in colorectal cancer. The technology performed with a high degree of accuracy and was able to robustly distinguish between cancer and NAM, NAM and adenoma and adenomas and cancers. Although promising, the number of adenomatous polyps assessed here was small, and clearly, this requires further analysis in a larger practice displayed as a simple visual readout providing data on predicted histological diagnosis and percentage chances that this is correct. However, the entire spectra are displayed, and the $600-900 \mathrm{~m} / \mathrm{z}$ range is shown in more detail, as this range is typically used in the multivariate analysis. The predominant phospholipid and triglyceride peaks can be visualized. G An adenoma snared during an in vivo assessment of the REIMS platform with representative anatomically discrete lipidomic data captured during hot snare deployment

patient population. It has also not assessed whether REIMS is able to detect a focus of cancer within a polyp, which is clearly the gold standard for any technology making an analysis of suspicious polyps. Sphingolipid metabolites have been analyzed by high-performance liquid chromatography (HPLC), and tubular adenomas have been demonstrated to have increased concentrations of ceramides compared to control samples. Ceramide, for example, is characterized by proapoptotic and anti-proliferative properties, and in keeping with the data presented here, its concentration is known to fall with the progression to cancer [16]. This suggests that these data are promising and that REIMS could be used as a tool for the rapid identification of unknown lesions, or for involved in vivo margin detection when electrocautery is deployed during EMR and ESD. 
REIMS also demonstrated significant promise in its ability to identify tumors of a poor prognostic phenotype. It was able to consistently identify histopathological features of poor prognosis (tumor budding, differentiation, LVI, EMVI and LN micrometastases). It is therefore possible that REIMS could chemically stage cancers in vivo and that this could be used as an adjunct to standard imaging technologies to improve preoperative staging and for stratifying neoadjuvant therapy. Clearly, we have not demonstrated a mechanistic link between each histopathological feature and target lipids, but this was not the aim of the current study. Moreover, we have not directly compared the performance of REIMS to existing staging methodologies such as MRI, which requires a prospective analysis in a larger patient cohort that we are currently undertaking. What this study demonstrates is that the chemistry of electrosurgical plumes contains rich and detailed information on lesions that are likely to confer a poor prognosis, and these data could therefore be used for stratifying surgical therapy (e.g., radical resection vs. endoluminal therapy and organ preservation). Further work is now needed to identify specific lipid targets and pathways that are regulated by each of these processes. Of specific interest, we were also able to clearly identify rectal samples previously treated with LCRT (Supp. Figure 2). This is not in itself a particular surprise, as radiotherapy will have a significant influence on cell membranes with a high turnover. However, it does suggest that REIMS is able to measure the impact of such an intervention in vivo. If it can be demonstrated that this can be achieved longitudinally, it may serve as a completely novel methodology for predicting complete pathological response in sensitive tumors or for identifying early recurrences.

A number of other optical spectroscopic techniques are being applied to endoscopic characterization of colonic lesions [17, 18]. An advantage of REIMS over existing optical spectroscopic strategies, which relay high-resolution information about lesion structure, is that REIMS provides information about tumor biology and chemistry. For some time, it has been known that increasingly aberrant lipid metabolism is associated with advancing neoplasia [19]. These changes are linked to increasing tumor invasiveness and metastatic potential. In this study, we have shown that by analyzing lipid ratios, REIMS correlates with a series of markers indicative of worsening pathogenicity and thus poorer prognosis in colorectal cancer. The REIMS method can do this in near real time, providing the endoscopist or surgeon with immediate information, which could help to inform decisions about subsequent treatment. This technology is being deployed in vivo and delivering reproducible data. More importantly, it can be deployed as an adjunct to any imaging technology, as the endoscope itself does not require any form of modification. Therefore, REIMS is a tool that can potentially chemically augment other tools deployed in a minimally invasive surgical environment for improving the accuracy of surgical or endoscopic decision-making.

In this analysis, we have gone beyond the ex vivo work to demonstrate a translational application of this precision medicine technology in a first in-man study of colonic polyps. We have successfully used this approach to record viable spectra from patients undergoing elective colonoscopy for polyps. Although this approach has yet to be used for polyp identification, we are able to obtain high-quality spectra that contain many of the metabolites identified from the ex vivo analysis (supp. Figure 3). From this dataset, we are able to generate spectra with an acceptable signal-tonoise ratio, with the level of detail necessary for multivariate analysis. This proof of concept suggests that endoscopic REIMS will be suitable for scaling into an in vivo clinical trial.

The authors acknowledge limitations with this pilot study. The sample size is small, and validation of our results in a larger patient cohort is needed. The exact mechanisms by which REIMS distinguishes clinically relevant variables are also not yet well understood. It is also possible that there is selection bias here as we were only collecting ex vivo specimens at surgery. Normal adjacent mucosa at $10 \mathrm{~cm}$ from the primary lesion was available for examination, as opposed to mucosa directly at the lesion margin, in order not to compromise the histopathological integrity of the surgical specimen. Our previous work, using a complementary mass spectrometry technique, suggests that there are metabolic differences between directly adjacent and 10-cm adjacent mucosa [15]. However, the aim of this feasibility study was to assess the diagnostic accuracy of REIMS in detecting cancer, for which $10-\mathrm{cm}$ adjacent mucosa is a suitable control. Energy is not always deployed in the endoscopic biopsy of polyps, and it is not always safe to deploy electrocautery. However, we believe that it is possible to minimize the amount of energy required to sample polyps so that it may be safely used, and these can also be assessed ex vivo after endoluminal capture. Irrespective of this, these data are promising and warrant further examination in the context of a larger-scale study. More importantly, these data will permit power calculations to be performed for future prospective work.

In conclusion, by augmenting optical endoscopic and radiological imaging techniques with information about tissue biochemistry, REIMS offers a new avenue in personalized oncological management through the real-time chemical analysis of the colorectal mucosal lipidome. Larger-scale studies of this technology are now ongoing to try to determine whether the data presented here can be scaled. 
Acknowledgments The authors would like to acknowledge the support of Waters Corporation and the help of Medwork in developing the modified endoscopic snare used in this study. We also thank Medical Illustration UK Ltd. for the provision of endoscopic photographs and video footage.

Funding The research was supported by the National Institute for Health Research (NIHR) Biomedical Research Centre based at Imperial College Healthcare National Health Service (NHS) Trust and Imperial College London. It was also funded by Bowel and Cancer Research and the European Research Council under European Research Council Consolidator Grant, Grant Agreement No: 617896.

\section{Compliance with ethical standards}

Disclosures James Alexander, Louise Gildea Julia Balog, Abigail Speller, James McKenzie, Laura Muirhead, Alasdair Scott, Christos Kontovounisios, Shanawaz Rasheed, Julian Teare, Jonathan Hoare, Kirill Veselkov, Robert Goldin, Paris Tekkis, James Kinross have no conflicting interests or financial ties to disclose. Ara Darzi, Jeremy Nicholson and Zoltan Takats perform consultancy work for Waters Corporation

Open Access This article is distributed under the terms of the Creative Commons Attribution 4.0 International License (http://crea tivecommons.org/licenses/by/4.0/), which permits unrestricted use, distribution, and reproduction in any medium, provided you give appropriate credit to the original author(s) and the source, provide a link to the Creative Commons license, and indicate if changes were made.

\section{References}

1. Mirnezami R, Nicholson J, Darzi A (2012) Preparing for precision medicine. N Engl J Med 366(6):489-491

2. Nicholson JK et al (2012) Metabolic phenotyping in clinical and surgical environments. Nature 491(7424):384-392

3. Gupta S (2015) Trouble in Paris (classification): polyp morphology is in the eye of the beholder. Am J Gastroenterol 110(1):188-191

4. Schafer KC et al (2011) Real time analysis of brain tissue by direct combination of ultrasonic surgical aspiration and sonic spray mass spectrometry. Anal Chem 83(20):7729-7735

5. Hinz KP et al (2011) Characterization of surgical aerosols by the compact single-particle mass spectrometer LAMPAS 3. Anal Bioanal Chem 401(10):3165-3172
6. Balog $\mathrm{J}$ et al (2010) Identification of biological tissues by rapid evaporative ionization mass spectrometry. Anal Chem 82(17):7343-7350

7. Balog $\mathbf{J}$ et al (2013) Intraoperative tissue identification using rapid evaporative ionization mass spectrometry. Sci Transl Med 5(194): 194ra93

8. Balog $\mathbf{J}$ et al (2015) In vivo endoscopic tissue identification by rapid evaporative ionization mass spectrometry (REIMS). Angew Chem Int Ed Engl 54(38):11059-11062

9. Shepherd M.B.L.P.Q.N.A (2014) Dataset for colorectal cancer histopathology reports (3rd edition). The Royal College of Pathologists

10. Veselkov KA et al (2014) Chemo-informatic strategy for imaging mass spectrometry-based hyperspectral profiling of lipid signatures in colorectal cancer. Proc Natl Acad Sci USA 111(3):1216-1221

11. Santos CR, Schulze A (2012) Lipid metabolism in cancer. FEBS J 279(15):2610-2623

12. Golf $\mathrm{O}$ et al (2015) Rapid evaporative ionization mass spectrometry imaging platform for direct mapping from bulk tissue and bacterial growth media. Anal Chem 87(5):2527-2534

13. Jimenez B et al (2013) 1H HR-MAS NMR spectroscopy of tumor-induced local metabolic "field-effects" enables colorectal cancer staging and prognostication. J Proteome Res 12(2):959-968

14. Mirnezami R et al (2014) Rapid diagnosis and staging of colorectal cancer via high-resolution magic angle spinning nuclear magnetic resonance (HR-MAS NMR) spectroscopy of intact tissue biopsies. Ann Surg 259(6):1138-1149

15. Mirnezami R et al (2014) Chemical mapping of the colorectal cancer microenvironment via MALDI imaging mass spectrometry (MALDI-MSI) reveals novel cancer-associated field effects. Mol Oncol 8(1):39-49

16. Kurek K et al (2015) Sphingolipid metabolism in colorectal adenomas varies depending on histological architecture of polyps and grade of nuclear dysplasia. Lipids 50(4):349-358

17. McGill SK et al (2013) Narrow band imaging to differentiate neoplastic and non-neoplastic colorectal polyps in real time: a meta-analysis of diagnostic operating characteristics. Gut 62(12):1704-1713

18. Kuiper $\mathrm{T}$ et al (2012) Feasibility and accuracy of confocal endomicroscopy in comparison with narrow-band imaging and chromoendoscopy for the differentiation of colorectal lesions. Am J Gastroenterol 107(4):543-550

19. Menendez JA, Lupu R (2007) Fatty acid synthase and the lipogenic phenotype in cancer pathogenesis. Nat Rev Cancer 7(10):763-777 